\title{
Editorial Note: Heterogeneous Knowledge Learning for Multimedia Analytics
}

Multimedia Tools and Applications gratefully acknowledges the editorial work of the scholars listed below on the special issue entitled, "Heterogeneous Knowledge Learning for Multimedia Analytics".

Of 41 papers submitted to this issue, 16 were eventually accepted after a stringent peer-review process.

\section{Dr. Ke Gao (Corresponding Guest Editor)}

Chinese Academy of Sciences, China

(kegao@ict.ac.cn)

\section{Dr. An-An Liu}

Tianjin University, China

(anan0422@gmail.com)

\section{Dr. Tom Yan}

University of Trento, Italy

( tom.yan.555@gmail.com)

\section{Dr. Mei Chen}

State University of New York, Albany

(meichen@albany.edu) 